\title{
The influence of $\theta$-function to the class of MWP operators
}

\author{
Ishak Altun, Gonca Durmaz
}

\begin{abstract}
In this work, taking into account the $\theta$-function, we present a general class of multivalued weakly Picard operators on complete metric space. We also provide an example showing that it includes some earlier classes as properly.
\end{abstract}

\section{INTRODUCTION}

One of the most important concept of metric fixed point theory is Multivalued Weakly Picard (shortly MWP) operator introduced by Rus [21] in 1991. Let $(X, d)$ be a metric space and $\mathcal{P}(X)$ be the family of all nonempty subsets of $X$. A multivalued mapping $T: X \rightarrow \mathcal{P}(X)$ is Multivalued Weakly Picard operator if there exists a sequence $\left\{x_{n}\right\}$ in $X$ such that $x_{n+1} \in T x_{n}$ for any initial point $x_{0}$, which converges to a fixed point of $T$. We shall denote the class of all MWP operators on $X$ by $\mathcal{M W P}(X)$. There are a lot of papers and results about MWP operators in the literature (see [17, 18, 19, 20]).

For the sake of completeness we recall some important concepts and results about multivalued mappings.

Let $(X, d)$ be a metric space. We denote by $\mathcal{C B}(X)$ the family of all nonempty closed and bounded subsets of $X$ and by $\mathcal{K}(X)$ the family of all nonempty compact subsets of $X$. Let $H$ be the Pompeiu-Hausdorff metric (see $[2,11])$ with respect to $d$, that is,

$$
H(A, B)=\max \left\{\sup _{x \in A} D(x, B), \sup _{y \in B} D(y, A)\right\},
$$

for every $A, B \in \mathcal{C B}(X)$, where $D(x, A)=\inf \{d(x, y): y \in A\}$. In 1969, Nadler [17] initiated the idea for multivalued contraction mapping and extended the Banach contraction principle to multivalued mappings and proved the following:

2010 Mathematics Subject Classification. Primary: 54H25; Secondary: 47H10.

Key words and phrases. Weakly Picard operator, multivalued mappings, $\theta$-contraction, fixed point.

Full paper. Received 28 January 2020, revised 9 April 2020, accepted 29 April 2020, available online 12 June 2020. 
Theorem 1. Let $(X, d)$ be a complete metric space and $T: X \rightarrow \mathcal{C B}(X)$ be multivalued mapping. If $T$ is a multivalued contraction, that is, there exists $\lambda \in[0,1)$ such that

$$
H(T x, T y) \leq \lambda d(x, y)
$$

for all $x, y \in X$, then there exists $z \in X$ such that $z \in T z$.

Later on, several researches were conducted on a variety of generalizations, extensions and applications of this result of Nadler (see [3, 8, 9, 13, 14, 16]). Furthermore, Berinde and Berinde [1] introduced the concepts of multivalued almost contraction and multivalued nonlinear almost contraction as follows: Let $(X, d)$ be a metric space and $T: X \rightarrow \mathcal{C B}(X)$ be a mapping. Then,

(i) $T$ is said to be a multivalued almost contraction if there exist two constants $\lambda \in(0,1)$ and $L \geq 1$ such that

$$
H(T x, T y) \leq \lambda d(x, y)+L D(y, T x)
$$

for all $x, y \in X$. We will denote the class of multivalued almost contractions on $X$ by $\mathcal{M A}(X)$.

(ii) $T$ is said to be a multivalued nonlinear almost contraction if there exists a constant $L \geq 0$ and a function $\varphi:[0, \infty) \rightarrow[0,1)$ satisfying

$$
\limsup _{t \rightarrow s^{+}} \varphi(t)<1, \forall s \geq 0,
$$

such that

$$
H(T x, T y) \leq \varphi(d(x, y)) d(x, y)+L D(y, T x),
$$

for all $x, y \in X$. We denote the class of all multivalued nonlinear almost contractions on $X$ by $\mathcal{M N} \mathcal{A}(X)$.

A function $\varphi:[0, \infty) \rightarrow[0,1)$ satisfying (1) is called Mizoguchi-Takahashi function ( as short $\mathcal{M T}$-function [7, 8, 22]) in the literature. Let's note, by the symmetry property of the metric, the above contractive conditions implicitly includes their dual ones. If $L=0$, then (2) turns to the famous Mizoguchi-Takahashi [16] contractive condition, which includes the multivalued contraction in sense of Nadler [17]. If we examine the proofs of Theorem 3 and Theorem 4 of [1], we can infer the following:

Theorem 2. If $(X, d)$ is a complete metric space, then

$$
\mathcal{M A}(X) \subseteq \mathcal{M N} \mathcal{A}(X) \subseteq \mathcal{M W \mathcal { P }}(X) .
$$

On the other hand, Jleli and Samet [12] presented an interesting generalization of the Banach contraction principle. They introduced a new type of contractive condition, which we shall call it as $\theta$-contraction. Now, we recall basic definitions, relevant notions and some related results concerning $\theta$-contraction. Let $\theta:(0, \infty) \rightarrow(1, \infty)$ be a function. Next we will consider the following properties for $\theta$ :

$\left(\theta_{1}\right) \theta$ is nondecreasing; 
$\left(\theta_{2}\right)$ For each sequence $\left\{t_{n}\right\} \subset(0, \infty), \lim _{n \rightarrow \infty} \theta\left(t_{n}\right)=1$ and $\lim _{n \rightarrow \infty} t_{n}=$ $0^{+}$are equivalent;

$\left(\theta_{3}\right)$ There exist $r \in(0,1)$ and $l \in(0, \infty]$ such that $\lim _{t \rightarrow 0^{+}} \frac{\theta(t)-1}{t^{r}}=l$;

$\left(\theta_{4}\right) \theta(\inf A)=\inf \theta(A)$ for all $A \subset(0, \infty)$ with $\inf A>0$.

We denote by $\Theta$ and $\Omega$ be the set of all functions $\theta$ satisfying $\left(\theta_{1}\right)-\left(\theta_{3}\right)$ and $\left(\theta_{1}\right)-\left(\theta_{4}\right)$, respectively. It is clear that $\Omega \subset \Theta$. Some examples of the functions belonging $\Omega$ are $\theta_{1}(t)=e^{\sqrt{t}}$ and $\theta_{2}(t)=e^{\sqrt{t e^{t}}}$. If we define

$$
\theta_{3}(t)=\left\{\begin{array}{cc}
e^{\sqrt{t}}, & t<1 \\
9, & t \geq 1
\end{array}\right.
$$

then, we can see $\theta_{3} \in \Theta \backslash \Omega$. Note that, if a function $\theta$ satisfies $\left(\theta_{1}\right)$, then it satisfies $\left(\theta_{4}\right)$ if and only if it is right continuous.

By considering the conditions $\left(\theta_{1}\right)-\left(\theta_{3}\right)$, Jleli and Samet [12] introduced the concept of $\theta$-contraction, which is more general than Banach contraction. Let $(X, d)$ be a metric space and $\theta \in \Theta$. A mapping $T: X \rightarrow X$ is said to be a $\theta$-contraction if there exists a constant $k \in[0,1)$ such that

$$
\theta(d(T x, T y)) \leq[\theta(d(x, y))]^{k},
$$

for all $x, y \in X$ with $d(T x, T y)>0$. As a real generalization of Banach contraction principle, Jleli and Samet proved that every $\theta$-contraction on a complete metric space has a unique fixed point. In addition, from $\left(\theta_{1}\right)$ and (3), it is easy to concluded that every $\theta$-contraction $T$ is a contractive mapping, i.e., $d(T x, T y)<d(x, y)$ for all $x, y \in X$ with $T x \neq T y$. Thus, every $\theta$-contraction mapping on a metric space is continuous.

Afterwards, many researches were conducted on a variety of generalizations, extensions and applications of the result of Jleli and Samet (See $[4,5,6,10,15])$. Hançer et al. [10] also extended the concept of $\theta$-contraction to multivalued case. Moreover in these directions, Durmaz and Altun [5] and Minak and Altun [15] presented the following concepts: Let $(X, d)$ be a metric space and $T: X \rightarrow \mathcal{C B}(X)$ be given a mapping. Then,

(i) $T$ is said to be a multivalued almost $\theta$-contraction with $\theta \in \Theta$ [5] if there exist two constants $k \in(0,1)$ and $\lambda \geq 0$ such that

$$
\theta(H(T x, T y)) \leq[\theta(d(x, y)+\lambda D(y, T x))]^{k},
$$

for all $x, y \in X$ with $H(T x, T y)>0$.

(ii) $T$ is said to be a multivalued nonlinear $\theta$-contraction with $\theta \in \Theta[15]$ if there exists a function $k:(0, \infty) \rightarrow[0,1)$ such that

$$
\lim \sup k(t)<1, \forall s \geq 0,
$$

satisfying

$$
\theta(H(T x, T y)) \leq[\theta(d(x, y))]^{k(d(x, y))},
$$

for all $x, y \in X$ with $H(T x, T y)>0$. 
We shall denote the class of all multivalued almost $\theta$-contractions with $\theta \in \Theta$ (resp. $\theta \in \Omega$ ) on $X$ by $\mathcal{M} \mathcal{A}_{\Theta}(X)$ (resp. $\mathcal{M} \mathcal{A}_{\Omega}(X)$ ) and the class of all multivalued nonlinear $\theta$-contractions with $\theta \in \Theta$ (resp. $\theta \in \Omega$ ) on $X$ by $\mathcal{M N}_{\Theta}(X)$ (resp. $\left.\mathcal{M N}_{\Omega}(X)\right)$. If we examine the proof of Theorem 2.1 in [5] and the proof of Theorem 8 in [15], we can infer the following theorems, respectively:

Theorem 3. If $(X, d)$ is a complete metric space, then

$$
\mathcal{M} \mathcal{A}_{\Omega}(X) \subseteq \mathcal{M W} \mathcal{P}(X) .
$$

Theorem 4. If $(X, d)$ is a complete metric space, then

$$
\mathcal{M N}_{\Omega}(X) \subseteq \mathcal{M W} \mathcal{P}(X) .
$$

We can see from the above definitions and theorems that if $(X, d)$ is a metric space, then

$$
\mathcal{M A}(X) \subseteq \mathcal{M} \mathcal{A}_{\Omega}(X) \subseteq \mathcal{M} \mathcal{A}_{\Theta}(X)
$$

and

$$
\mathcal{M N}(X) \subseteq \mathcal{M N}_{\Omega}(X) \subseteq \mathcal{M N}_{\Theta}(X)
$$

and further if $(X, d)$ is complete metric space, then

$$
\mathcal{M A}_{\Omega}(X) \cup \mathcal{M} \mathcal{N}_{\Omega}(X) \subseteq \mathcal{M W \mathcal { P }}(X) .
$$

However, Example 1 of [15] shows that, even if $(X, d)$ is a complete metric space, then

$$
\mathcal{M A}_{\Theta}(X) \nsubseteq \mathcal{M \mathcal { W }} \mathcal{P}(X) \text { and } \mathcal{M N}_{\Theta}(X) \nsubseteq \mathcal{M} \mathcal{W} \mathcal{P}(X) .
$$

In this paper, we present a general class of MWP operators on a complete metric space $(X, d)$ which includes the classes $\mathcal{M N} \mathcal{A}(X), \mathcal{M} \mathcal{A}_{\Omega}(X)$ and $\mathcal{M N}_{\Omega}(X)$.

\section{The RESUlts}

Definition 1. Let $(X, d)$ be a metric space, $T: X \rightarrow \mathcal{C B}(X)$ be a mapping. We say that $T$ is a multivalued nonlinear almost $\theta$-contraction with $\theta \in \Theta$ if there exists a constant $\lambda \geq 0$ and a function $k:(0, \infty) \rightarrow[0,1)$ such that

$$
\limsup _{t \rightarrow s^{+}} k(t)<1, \forall s \geq 0,
$$

satisfying

$$
\theta(H(T x, T y)) \leq[\theta(d(x, y)+\lambda D(y, T x))]^{k(d(x, y))},
$$

for all $x, y \in X$ with $H(T x, T y)>0$.

We shall denote the class of all multivalued nonlinear almost $\theta$-contractions with $\theta \in \Theta\left(\right.$ resp. $\theta \in \Omega$ ) on $X$ by $\mathcal{M N} \mathcal{A}_{\Theta}(X)$ (resp. $\left.\mathcal{M N} \mathcal{A}_{\Omega}(X)\right)$. It is clear that

$$
\mathcal{M A}_{\Theta}(X) \cup \mathcal{M} \mathcal{N}_{\Theta}(X) \cup \mathcal{M N} \mathcal{A}(X) \subseteq \mathcal{M N} \mathcal{A}_{\Theta}(X)
$$


Now we give our main result, which presents a general class of MWP operators on complete metric space.

Theorem 5. If $(X, d)$ is a complete metric space, then $\mathcal{M N} \mathcal{A}_{\Omega}(X) \subseteq$ $\mathcal{M W P}(X)$.

Kanıt. Let $(X, d)$ be a complete metric space and $T \in \mathcal{M N} \mathcal{A}_{\Omega}(X)$. Define a set $X^{*}=\{x \in X: D(x, T x)>0\}$. Let $x_{0} \in X \backslash X^{*}$ be an arbitrary point, then $x_{0}$ is a fixed point of $T$ and also the sequence $\left\{x_{n}\right\}=\left\{x_{0}, x_{0}, x_{0}, \ldots\right\}$ converges to $x_{0}$ which satisfies $x_{n+1} \in T x_{n}$. Now let $x_{0} \in X^{*}$ and choose $x_{1} \in T x_{0}$. If $x_{1} \in X \backslash X^{*}$, then $x_{1}$ is a fixed point of $T$ and so we can construct a Picard sequence which converges to $x_{1}$. Suppose $x_{1} \in X^{*}$, then we have $0<D\left(x_{1}, T x_{1}\right) \leq H\left(T x_{0}, T x_{1}\right)$ and so from $\left(\theta_{1}\right)$, we obtain

$$
\theta\left(D\left(x_{1}, T x_{1}\right)\right) \leq \theta\left(H\left(T x_{0}, T x_{1}\right)\right) .
$$

From (4), we can write that

$$
\begin{aligned}
\theta\left(D\left(x_{1}, T x_{1}\right)\right) & \leq \theta\left(H\left(T x_{0}, T x_{1}\right)\right) \\
& \leq\left[\theta\left(d\left(x_{0}, x_{1}\right)+\lambda D\left(x_{1}, T x_{0}\right)\right)\right]^{k\left(d\left(x_{0}, x_{1}\right)\right)} \\
& =\left[\theta\left(d\left(x_{0}, x_{1}\right)\right)\right]^{k\left(d\left(x_{0}, x_{1}\right)\right)} .
\end{aligned}
$$

From $\left(\theta_{4}\right)$, we can write

$$
\theta\left(D\left(x_{1}, T x_{1}\right)\right)=\inf _{y \in T x_{1}} \theta\left(d\left(x_{1}, y\right)\right)
$$

and so from (5) we have

$$
\begin{aligned}
\inf _{y \in T x_{1}} \theta\left(d\left(x_{1}, y\right)\right) & \leq\left[\theta\left(d\left(x_{0}, x_{1}\right)\right)\right]^{k\left(d\left(x_{0}, x_{1}\right)\right)} \\
& <\left[\theta\left(d\left(x_{0}, x_{1}\right)\right)\right]^{\frac{k\left(d\left(x_{0}, x_{1}\right)\right)}{2}} .
\end{aligned}
$$

Then, from (6) there exists $x_{2} \in T x_{1}$ such that

$$
\theta\left(d\left(x_{1}, x_{2}\right)\right) \leq\left[\theta\left(d\left(x_{0}, x_{1}\right)\right)\right]^{\frac{k\left(d\left(x_{0}, x_{1}\right)\right)}{2}} .
$$

If $x_{2} \in X \backslash X^{*}$, then $x_{2}$ is a fixed point of $T$. Otherwise, by the same way, we can find $x_{3} \in T x_{2}$ such that

$$
\theta\left(d\left(x_{2}, x_{3}\right)\right) \leq\left[\theta\left(d\left(x_{1}, x_{2}\right)\right)\right]^{\frac{k\left(d\left(x_{1}, x_{2}\right)\right)}{2}} .
$$

Therefore, continuing recursively, we can obtain a sequence $\left\{x_{n}\right\}$ in $X^{*}$ such that $x_{n+1} \in T x_{n}$ and

$$
\theta\left(d\left(x_{n}, x_{n+1}\right)\right) \leq\left[\theta\left(d\left(x_{n-1}, x_{n}\right)\right)\right]^{\frac{k d\left(x_{n-1}, x_{n}\right)}{2}}
$$

for all $n \in \mathbb{N}$. Thus the sequence $\left\{d\left(x_{n}, x_{n+1}\right)\right\}$ is decreasing and hence convergent. From (7), there exists $b \in(0,1)$ and $n_{0} \in \mathbb{N}$ such that $k\left(d\left(x_{n}, x_{n+1}\right)\right)$ $<b$ for all $n \geq n_{0}$. Thus, we obtain for all $n \geq n_{0}$ the following inequalities: 


$$
\begin{aligned}
1< & \theta\left(d\left(x_{n}, x_{n+1}\right)\right) \\
\leq & {\left[\theta\left(d\left(x_{n-1}, x_{n}\right)\right)\right]^{k\left(d\left(x_{n-1}, x_{n}\right)\right)} } \\
\leq & {\left[\theta\left(d\left(x_{n-2}, x_{n-1}\right)\right)\right]^{k\left(d\left(x_{n-1}, x_{n}\right)\right) k\left(d\left(x_{n-1}, x_{n}\right)\right)} } \\
& \vdots \\
\leq & {\left[\theta\left(d\left(x_{0}, x_{1}\right)\right)\right]^{k\left(d\left(x_{0}, x_{1}\right)\right) \cdots k\left(d\left(x_{n-1}, x_{n}\right)\right) k\left(d\left(x_{n-1}, x_{n}\right)\right)} } \\
= & {\left[\theta\left(d\left(x_{0}, x_{1}\right)\right)\right]^{k\left(d\left(x_{0}, x_{1}\right)\right) \cdots k\left(d\left(x_{n_{0}-1}, x_{n_{0}}\right)\right) k\left(d\left(x_{n_{0}}, x_{n_{0}+1}\right)\right) \cdots k\left(d\left(x_{n-1}, x_{n}\right)\right) k\left(d\left(x_{n-1}, x_{n}\right)\right)} } \\
\leq & {\left[\theta\left(d\left(x_{0}, x_{1}\right)\right)\right]^{k\left(d\left(x_{n_{0}}, x_{n_{0}+1}\right)\right) \cdots k\left(d\left(x_{n-1}, x_{n}\right)\right) k\left(d\left(x_{n-1}, x_{n}\right)\right)} } \\
\leq & {\left[\theta\left(d\left(x_{0}, x_{1}\right)\right)\right]^{b^{\left(n-n_{0}\right)}} . }
\end{aligned}
$$

Thus, we obtain

$$
1<\theta\left(d\left(x_{n}, x_{n+1}\right)\right) \leq\left[\theta\left(d\left(x_{0}, x_{1}\right)\right)\right]^{b^{\left(n-n_{0}\right)}}
$$

for all $n \geq n_{0}$. Letting $n \rightarrow \infty$ in (8), we obtain

$$
\lim _{n \rightarrow \infty} \theta\left(d\left(x_{n}, x_{n+1}\right)\right)=1 \text {. }
$$

From $\left(\theta_{2}\right), \lim _{n \rightarrow \infty} d\left(x_{n}, x_{n+1}\right)=0^{+}$and so from $\left(\theta_{3}\right)$ there exist $r \in(0,1)$ and $l \in(0, \infty]$ such that

$$
\lim _{n \rightarrow \infty} \frac{\theta\left(d\left(x_{n}, x_{n+1}\right)\right)-1}{\left[d\left(x_{n}, x_{n+1}\right)\right]^{r}}=l .
$$

Suppose that $l<\infty$. In this case, let $B=\frac{l}{2}>0$. From the definition of the limit, there exists $n_{0} \in \mathbb{N}$ such that, for all $n \geq n_{0}$,

$$
\left|\frac{\theta\left(d\left(x_{n}, x_{n+1}\right)\right)-1}{\left[d\left(x_{n}, x_{n+1}\right)\right]^{r}}-l\right| \leq B .
$$

This implies that, for all $n \geq n_{0}$,

$$
\frac{\theta\left(d\left(x_{n}, x_{n+1}\right)\right)-1}{\left[d\left(x_{n}, x_{n+1}\right)\right]^{r}} \geq l-B=B .
$$

Then, for all $n \geq n_{0}$,

$$
n\left[d\left(x_{n}, x_{n+1}\right)\right]^{r} \leq A n\left[\theta\left(d\left(x_{n}, x_{n+1}\right)\right)-1\right],
$$

where $A=1 / B$.

Suppose now that $l=\infty$. Let $B>0$ be an arbitrary positive number. From the definition of the limit, there exists $n_{0} \in \mathbb{N}$ such that, for all $n \geq n_{0}$,

$$
\frac{\theta\left(d\left(x_{n}, x_{n+1}\right)\right)-1}{\left[d\left(x_{n}, x_{n+1}\right)\right]^{r}} \geq B .
$$

This implies that, for all $n \geq n_{0}$,

$$
n\left[d\left(x_{n}, x_{n+1}\right)\right]^{r} \leq A n\left[\theta\left(d\left(x_{n}, x_{n+1}\right)\right)-1\right],
$$

where $A=1 / B$. 
Thus, in all cases, there exist $A>0$ and $n_{0} \in \mathbb{N}$ such that, for all $n \geq n_{0}$,

$$
n\left[d\left(x_{n}, x_{n+1}\right)\right]^{r} \leq A n\left[\theta\left(d\left(x_{n}, x_{n+1}\right)\right)-1\right] .
$$

Using (8), we obtain, for all $n \geq n_{0}$,

$$
n\left[d\left(x_{n}, x_{n+1}\right)\right]^{r} \leq A n\left[\left[\theta\left(d\left(x_{0}, x_{1}\right)\right)\right]^{b^{\left(n-n_{0}\right)}}-1\right] .
$$

Letting $n \rightarrow \infty$ in the above inequality, we obtain

$$
\lim _{n \rightarrow \infty} n\left[d\left(x_{n}, x_{n+1}\right)\right]^{r}=0 .
$$

Thus, there exits $n_{1} \in \mathbb{N}$ such that $n\left[d\left(x_{n}, x_{n+1}\right)\right]^{r} \leq 1$ for all $n \geq n_{1}$. So, we have, for all $n \geq n_{1}$

$$
d\left(x_{n}, x_{n+1}\right) \leq \frac{1}{n^{1 / r}} .
$$

In order to show that $\left\{x_{n}\right\}$ is a Cauchy sequence, consider $m, n \in \mathbb{N}$ such that $m>n \geq n_{1}$. Using the triangular inequality for the metric and from (10), we have

$$
\begin{aligned}
d\left(x_{n}, x_{m}\right) & \leq d\left(x_{n}, x_{n+1}\right)+d\left(x_{n+1}, x_{n+2}\right)+\cdots+d\left(x_{m-1}, x_{m}\right) \\
& =\sum_{i=n}^{m-1} d\left(x_{i}, x_{i+1}\right) \leq \sum_{i=n}^{\infty} d\left(x_{i}, x_{i+1}\right) \leq \sum_{i=n}^{\infty} \frac{1}{i^{1 / r}} .
\end{aligned}
$$

By the convergence of the series $\sum_{i=1}^{\infty} \frac{1}{i^{1 / r}}$, letting to limit $n \rightarrow \infty$, we get $d\left(x_{n}, x_{m}\right) \rightarrow 0$. This yields that $\left\{x_{n}\right\}$ is a Cauchy sequence in $(X, d)$. Since $(X, d)$ is a complete metric space, the sequence $\left\{x_{n}\right\}$ converges to some point $z \in X$. From $\left(\theta_{1}\right)$ and (4), for all $x, y \in X$ with $H(T x, T y)>0$, we get

$$
H(T x, T y)<d(x, y)+\lambda D(y, T x),
$$

and so

$$
H(T x, T y) \leq d(x, y)+\lambda D(y, T x),
$$

for all $x, y \in X$. Then

$$
\begin{aligned}
D\left(x_{n+1}, T z\right) & \leq H\left(T x_{n}, T z\right) \\
& \leq d\left(x_{n}, z\right)+\lambda D\left(z, T x_{n}\right) \\
& \leq d\left(x_{n}, z\right)+\lambda d\left(z, x_{n+1}\right) .
\end{aligned}
$$

Passing to limit $n \rightarrow \infty$ in the above, we obtain $D(z, T z)=0$. Thus, we get $z \in T z$. Therefore $T \in \mathcal{M} \mathcal{W} \mathcal{P}(X)$.

Now, we give a significant example showing that $T \in \mathcal{M W P}(X)$, since $T \in \mathcal{M N} \mathcal{A}_{\Omega}(X)$ when $(X, d)$ is a complete metric space. However, $T \notin$ $\mathcal{M N} \mathcal{A}(X) \cup \mathcal{M} \mathcal{N}_{\Omega}(X)$. 
Example 1. Consider the complete metric space $(X, d)$, where $X=\left\{\frac{1}{2^{n}}\right.$ : $n \in \mathbb{N}\} \cup\{0\}$ and

$$
d(x, y)=\left\{\begin{array}{cc}
0, & x=y \\
\max \{x, y\}, & x \neq y .
\end{array}\right.
$$

Define a mapping $T: X \rightarrow \mathcal{C B}(X)$ by

$$
T x=\left\{\begin{array}{cl}
\{x\}, & x \in\{0,1\} \\
\left\{\frac{1}{2^{n+1}}, \frac{1}{2^{n+2}}, \cdots\right\}, & x=\frac{1}{2^{n}}, \quad n \in \mathbb{N}, n>1 .
\end{array}\right.
$$

We claim that $T \in \mathcal{M N} \mathcal{A}_{\Omega}(X)$ with $\theta(t)=e^{\sqrt{t e^{t}}}, \lambda=\frac{1}{2}$ and $k:(0, \infty) \rightarrow$ $[0,1)$ defined by

$$
k(t)=\left\{\begin{array}{cl}
e^{-\frac{1}{2^{n+2}}}, & \text { if } t=\frac{1}{2^{n}} \text { for } n \in \mathbb{N}, \\
0, & \text { otherwise. }
\end{array}\right.
$$

It is clear that $\limsup _{t \rightarrow s^{+}} k(t)=0<1$ for all $s \in[0, \infty)$. Observe that taking $\theta(t)=e^{\sqrt{t e^{t}}}$ and $\lambda=\frac{1}{2}$ the contractive condition (4) turns to

$$
\frac{H(T x, T y) e^{H(T x, T y)-d(x, y)-\lambda \min \{d(y, T x), d(x, T y)\}}}{d(x, y)+\lambda \min \{d(y, T x), d(x, T y)\}} \leq[k(d(x, y))]^{2} .
$$

for all $x, y \in X$ with $H(T x, T y)>0$. Now we consider the following cases: for the brevity we will assign the left side of (11) as $A(x, y)$. Also without lost of generality we assume $x>y$ in all cases.

Case 1. Let $x=\frac{1}{2^{n}}$ and $y=\frac{1}{2^{m}}$ with $m>n>1$, then

$$
A(x, y)=\frac{\frac{1}{2^{n+1}}}{\frac{1}{2^{n}}} e^{-\frac{1}{2^{n+1}}}=\frac{1}{2} e^{-\frac{1}{2^{n+1}}} \leq k^{2}\left(\frac{1}{2^{n}}\right)=k^{2}(d(x, y)),
$$

Case 2. Let $x=\frac{1}{n}, n>1$ and $y=0$, then

$$
A(x, y)=\frac{\frac{1}{2^{n+1}}}{\frac{1}{2^{n}}} e^{-\frac{1}{2^{n+1}}}=\frac{1}{2} e^{-\frac{1}{2^{n+1}}} \leq k^{2}\left(\frac{1}{2^{n}}\right)=k^{2}(d(x, y)),
$$

Case 3. Let $x=1$ and $y=0$, then

$$
A(x, y)=\frac{1}{1+\frac{1}{2}} e^{-\frac{1}{2}}=\frac{2}{3} e^{-\frac{1}{2}} \leq e^{-\frac{1}{2}}=k^{2}(1)=k^{2}(d(x, y)) .
$$

Case 4. If $x=\frac{1}{n}, n>1$ and $y=1$, then

$$
A(x, y)=\frac{1}{1+\frac{1}{2}} e^{-\frac{1}{2}}=\frac{2}{3} e^{-\frac{1}{2}} \leq e^{-\frac{1}{2}}=k^{2}(1)=k^{2}(d(x, y)) .
$$

This shows that $T \in \mathcal{M N} \mathcal{A}_{\Omega}(X)$. Also since $(X, d)$ is complete metric space, then by Theorem $5, T \in \mathcal{M W \mathcal { P }}(X)$. 
On the other hand, since $H(T 0, T 1)=1=d(0,1)$, then for all $\theta \in \Omega$ and for all $k:(0, \infty) \rightarrow[0,1)$ satisfying inequality $(1)$, we have

$$
\theta(H(T 0, T 1))=\theta(1)>\theta(1)^{k(1)}=\theta(d(0,1))^{k(d(0,1))} .
$$

Therefore, $T \notin \mathcal{M N}_{\Omega}(X)$.

The following result is interested in the mapping $T: X \rightarrow \mathcal{K}(X)$. Here, we can remove the condition $\left(\theta_{4}\right)$ on the function $\theta$. For this, we will use that if $A$ is compact subset of a metric space $(X, d)$, then for every $x \in X$ there exists $a \in A$ such that $d(x, a)=d(x, A)$.

Theorem 6. Let $(X, d)$ be a complete metric space and $T: X \rightarrow \mathcal{K}(X)$ be a mapping. If $T \in \mathcal{M N} \mathcal{A}_{\Theta}(X)$, then $T \in \mathcal{M W \mathcal { P }}(X)$.

Kanıt. As in proof of Theorem 5, we get

$$
\begin{aligned}
\theta\left(D\left(x_{1}, T x_{1}\right)\right) & \leq \theta\left(H\left(T x_{0}, T x_{1}\right)\right) \\
& \leq\left[\theta\left(d\left(x_{0}, x_{1}\right)+\lambda D\left(x_{1}, T x_{0}\right)\right)\right]^{k\left(d\left(x_{0}, x_{1}\right)\right)} \\
& \leq\left[\theta\left(d\left(x_{1}, x_{0}\right)\right)\right]^{k\left(d\left(x_{0}, x_{1}\right)\right)} .
\end{aligned}
$$

Since $T x_{1}$ is compact, there exists $x_{2} \in T x_{1}$ such that $d\left(x_{1}, x_{2}\right)=d\left(x_{1}, T x_{1}\right)$. From (12),

$$
\begin{aligned}
\theta\left(d\left(x_{1}, x_{2}\right)\right) & \leq \theta\left(H\left(T x_{0}, T x_{1}\right)\right) \\
& \leq\left[\theta\left(d\left(x_{0}, x_{1}\right)+\lambda D\left(x_{1}, T x_{0}\right)\right)\right]^{k\left(d\left(x_{0}, x_{1}\right)\right)} \\
& <\left[\theta\left(d\left(x_{1}, x_{0}\right)\right)\right]^{k\left(d\left(x_{0}, x_{1}\right)\right)} .
\end{aligned}
$$

By induction, we obtain a sequence $\left\{x_{n}\right\}$ in $X^{*}$ with the property that $x_{n+1} \in T x_{n}$, and

$$
\theta\left(d\left(x_{n}, x_{n+1}\right)\right) \leq\left[\theta\left(d\left(x_{n-1}, x_{n}\right)\right)\right]^{k\left(d\left(x_{n-1}, x_{n}\right)\right)}<\theta\left(d\left(x_{n-1}, x_{n}\right)\right),
$$

for all $n \in \mathbb{N}$.

The rest of the proof can be completed as in the proof of Theorem 5 .

\section{ACKnowledgement}

The authors are thankful to the referee for making valuable suggestions leading to the better presentations of the paper.

\section{REFERENCES}

[1] M. Berinde and V. Berinde, On a general class of multi-valued weakly Picard mappings, Journal of Mathematical Analysis and Applications, 326 (2) (2007), 772-782.

[2] V. Berinde and M. Păcurar, The role of the Pompeiu-Hausdorff metric in fixed point theory, Creative Mathematics and Informatics, 22 (2013), 35-42.

[3] Lj. B. Ćirić, Multi-valued nonlinear contraction mappings, Nonlinear Analysis, 71 (2009), 2716-2723. 
[4] G. Durmaz, Some theorems for a new type of multivalued contractive maps on metric space, Turkish Journal of Mathematics, 41 (4) (2017), 1092-1100.

[5] G. Durmaz and I. Altun, A new perspective for multivalued weakly picard operators, Publications de l'Institut Mathématique, 101 (115) (2017), 197-204.

[6] G. Durmaz and I. Altun, On nonlinear set-valued $\theta$-contractions, Bulletin of the Malaysian Mathematical Sciences Society, 43 (2020), 389-402.

[7] W-S. Du, On coincidence point and fixed point theorems for nonlinear multivaled maps, Topology and its Applications, 159 (1) (2012), 49-56.

[8] W-S. Du, Some new results and generalizations in metric fixed point theory, Nonlinear Analysis: Theory, Methods and Applications, 73 (2010), 1439-1446.

[9] Y. Feng and S. Liu, Fixed point theorems for multi-valued contractive mappings and multi-valued Caristi type mappings, Journal of Mathematical Analysis and Applications, 317 (1) (2006), 103-112.

[10] H. A. Hançer, G. Mınak and I. Altun, On a broad category of multivalued weakly Picard operators, Fixed Point Theory, 18 (1) (2017), 229-336.

[11] V. I. Istrățescu, Fixed Point Theory an Introduction, Dordrecht, D. Reidel Publishing Company, 1981.

[12] M. Jleli and B. Samet, A new generalization of the Banach contraction principle, Journal of Inequalities and Applications, 2014 (2014), ArticleID 38, 8 pages.

[13] T. Kamran and Q. Kiran, Fixed point theorems for multi-valued mappings obtained by altering distances, Mathematical and Computer Modelling, 54 (2011), 2772-2777.

[14] D. Klim and D. Wardowski, Fixed point theorems for set-valued contractions in complete metric spaces, Journal of Mathematical Analysis and Applications, 334 (1) (2007), 132-139.

[15] G. Minak and I. Altun, Overall approach to Mizoguchi-Takahashi type fixed point results, Turkish Journal of Mathematics, 40 (4) (2016), 895-904.

[16] N. Mizoguchi and W. Takahashi, Fixed point theorems for multivalued mappings on complete metric spaces, Journal of Mathematical Analysis and Applications, 141 (1) (1989), 177-188.

[17] S.B. Nadler, Multi-valued contraction mappings, Pacific Journal of Mathematics, 30 (1969), 475-488.

[18] A. Petruşel, On Frigon-Granas-type multifunctions, Nonlinear Analysis Forum, 7 (2002), 113-121.

[19] S. Reich, Fixed points of contractive functions, Bollettino dell'Unione Mathematica Italiana, 5 (1972), 26-42.

[20] I. A. Rus, Basic problems of the metric fixed point theory revisited (II), Studia Universitatis Babeş-Bolyai Mathematica, 36 (1991), 81-99.

[21] I. A. Rus, A. Petrusel, A. Sintamarian, Data dependence of the fixed point set of some multi-valued weakly Picard operators, Nonlinear Analysi, 52 (8) (2003) 1947-1959.

[22] T. Suzuki, Mizoguchi-Takahashi's fixed point theorem is a real generalization of Nadler's, Journal of Mathematical Analysis and Applications, 340 (1) (2008), 752-755. 


\section{Ishak Altun}

Department of Mathematics

Faculty of Science and Arts

KirikKale UNIVERSITY

71450 YaHSIHAN

KIRIKKALE

TURKEY

E-mail address: ishakaltun@yahoo.com

\section{Gonca Durmaz}

Department of Mathematics

FACUlTy OF SCIENCE

ÇANKIRI Karatekín University

18100 ÇANKIRI

TURKEY

E-mail address: gncmatematik@hotmail.com 